\title{
Liberals and Conservatives are Similarly Motivated to Avoid Exposure to One Another's Opinions
}

\author{
Jeremy A. Frimer, University of Winnipeg \\ Linda J. Skitka, \& Matt Motyl, University of Illinois, Chicago
}

May be cited as:

Frimer, J. , Skitka, L. J., \& Motyl, M. (2017). Liberals and conservatives are similarly motivated to avoid exposure to one another's opinions. Journal of Experimental Social Psychology, $72,1-12$.

Corresponding Author: Jeremy A. Frimer, Department of Psychology, University of Winnipeg,

515 Portage Avenue, Winnipeg MB, Canada, R3B 2E9, j.frimer@uwinnipeg.ca

Acknowledgements: This work was supported by a grant from the Social Sciences and

Humanities Research Council of Canada to J. A. Frimer [grant number 435-2013-0589]. 


\begin{abstract}
Ideologically committed people are similarly motivated to avoid ideologically crosscutting information. Although some previous research has found that political conservatives may be more prone to selective exposure than liberals are, we find similar selective exposure motives on the political left and right across a variety of issues. The majority of people on both sides of the same-sex marriage debate willingly gave up a chance to win money to avoid hearing from the other side (Study 1). When thinking back to the 2012 U.S. Presidential election (Study 2), ahead to upcoming elections in the U.S. and Canada (Study 3), and about a range of other Culture War issues (Study 4), liberals and conservatives reported similar aversion toward learning about the views of their ideological opponents. Their lack of interest was not due to already being informed about the other side or attributable election fatigue. Rather, people on both sides indicated that they anticipated that hearing from the other side would induce cognitive dissonance (e.g., require effort, cause frustration) and undermine a sense of shared reality with the person expressing disparate views (e.g., damage the relationship; Study 5). A high-powered meta-analysis of our data sets $(N=2417)$ did not detect a difference in the intensity of liberals' $(d=0.63)$ and conservatives' $(d=0.58)$ desires to remain in their respective ideological bubbles.
\end{abstract}

Keywords: selective exposure; confirmation bias; motivation; liberals and conservatives; ideological symmetry 


\section{Liberals and Conservatives are Similarly Motivated to Avoid Exposure to One Another's Opinions}

Only rarely do people dispassionately approach socio-political matters, such as whether abortion or owning automatic weapons should be permitted or limited. More often, people seem to gather, scrutinize, interpret, and remember information in a manner that confirms their preexisting opinions (see Kunda, 1990; Nickerson, 1998; and, Smith, Fabrigar, \& Norris, 2008, for reviews). A meta-analysis (Hart et al. 2009) attested to the prevalence of the confirmation/congeniality bias, with an average effect size of $d=0.36(d=0.46$ for political issues). At least three basic processes work in tandem to create a confirmation bias: (a) people selectively expose themselves to belief-confirming information, (b) people interpret information that is already in front of them in a belief-confirming manner, and (c) people remember information that confirms their beliefs. In this paper, we focus on one specific aspect of selective exposure, namely the motivation to avoid crosscutting information. And we investigate whether people on the political right and left are equally or differentially motivated to remain in their ideological bubble.

\section{Selective Exposure}

People tend to selectively expose themselves to belief-confirming information for at least two reasons. First, information that conflicts with one's own beliefs creates cognitive dissonance and feelings of personal discomfort (Festinger, 1957). This personal discomfort thesis aligns with the well-supported notion that selective exposure is a form of self-defense against feeling threatened (Webb, Chang, \& Benn, 2013; Hart et al., 2009). Selective exposure may also have interpersonal origins. According to the theory of shared reality (Echterhoff, Higgins, \& Levine, 
2009), people have a fundamental need to feel mental synchrony with others. Achieving a shared sense of reality requires that two or more people hold beliefs in common — and that they communicate their beliefs to one another. Seeking out information from like-minded others could satisfy this fundamental need and avoiding information from unlike-minded others could undermine this fundamental need. Liberals and conservatives may both engage in selective exposure to avoid cognitive dissonance and satisfy the need for a shared reality. But whether they do so to the same degree remains an unresolved matter.

\section{Is Selective Exposure Ideologically Symmetric? Mixed Evidence}

People on the political left tend to value a more liberal, "loose", egalitarian social structure, whereas people on the political right tend to value a more conservative, "tight", hierarchical social structure (Harrington \& Gelfand, 2004). Are people on the political right more motivated to remain ignorant of the lefts' views than vice versa?

Several theories and studies suggest that conservatives may be more prone to selective exposure than are liberals. System justification theory, social dominance theory, and right wing authoritarianism characterize liberals as thoughtful, tolerant of differing opinions, and openminded, and conservatives as fearful, prejudiced, and close-minded. For example, liberals seem to be more open to new experiences (e.g., McCrae, 1996) and are more analytic in their thinking style (Talhelm et al., 2015). In contrast, conservatives may be more prejudiced against and violent towards outgroups (Sibley \& Duckitt, 2008; Vail \& Motyl, 2010), dogmatic (Jost, Glaser, Kruglanski, \& Sulloway, 2003), and have a stronger need to reduce uncertainty and threat (Jost, Nosek, \& Gosling 2008). According to system justification theory, these basic psychological differences cause conservatives (relative to liberals) to more strongly endorse a rigid, hierarchical social system that is intolerant of dissenting views. This may mean that 
conservatives are more likely to work harder to avoid exposure to liberals' views—which conservatives perceive to be dissenting views - than vice versa.

On the question of whether selective exposure is ideologically symmetric, extant research has yielded mixed findings. Four studies found that conservatives are more prone to selective exposure than are liberals. First, Lazarsfeld, Berelson, and Gaudet (1944) found that Republicans read primarily pro-Republican media in the 1940 US Presidential election campaign season (Roosevelt vs. Willkie), whereas Democrats consumed equal amounts of pro-Republican and pro-Democratic media. Second, Nam, Jost, and van Bavel (2013) found that, when directly asked, liberals were more likely than conservatives to be willing to write a counter-ideological essay. Third, Iyengar, Hahn, Krosnick, and Walker (2008) found that during the 2000 US Presidential election campaign season (Bush vs. Gore), Republicans were more prone to selectively expose themselves to information about their preferred (Republican) candidate and to avoid information about their non-preferred (Democratic) candidate than were Democrats. And fourth, Barberá, Jost, Nagler, Tucker, and Bonneau (2015) found that, on Twitter, conservatives were less likely to retweet posts written by ideological opponents than were liberals (although, it is unclear whether they actually consumed the news contained in the links in those posts, or just shared them without reading their content).

One of the four studies just described, finding that conservatives are more prone to selective exposure, have been the subject of conceptual re-analyses. Specifically, Sears and Freedman (1967) pointed out that in the Lazarsfeld et al. (1944) study, the Republican Party spent more than twice as much as the Democratic Party in the 1940 electoral campaign (Overack, 1941), resulting in a greater availability of pro-Republican media to the public. That is, Republicans may have consumed more pro-Republican media simply because more Republican 
media was available. When taking into consideration the amount of available information, Sears and Freedman (1967) concluded that it was the Democratic Party members who were more prone to selective exposure in the 1940 electoral campaign than the Republicans (see p. 200). This follow-up analysis highlights the importance of leveling the contextual playing field before drawing inferences about ideological (a)symmetries.

Other studies have not shown that liberals are more prone to selective exposure effects than conservatives. Two studies show no differences (Iyengar \& Hahn, 2009; Stroud, 2008), and one, attempting to replicate the Nam et al. (2013) findings, failed to do so (Collins, Crawford, \& Brandt, 2015). One recent analysis of Facebook behaviors has shown the reverse effect: conservatives tended to click on and share ideologically crosscutting posts more than liberals did (Bakshy, Messing, \& Adamic 2015), meaning that liberals were more prone to selective exposure on Facebook. In sum, the scientific record regarding whether liberals or conservatives are more prone to selective exposure is mixed and inconclusive. Some studies find that conservatives are more prone to this bias, others find that liberals are more prone, and still others find no difference.

\section{Why are the Findings Mixed?}

Why has the growing literature on the ideological (a)symmetry of selective exposure produced mixed results? We suggest that study designs have varied in how well they actually measured selective exposure. Recall that in the Lazarsfeld et al. (1944) study, for instance, the amount of information available to the public was ideologically asymmetric-more proRepublican than Democratic information was available. This asymmetry in the availability of information may have explained why Republicans consumed more ideologically congenial information than did Democrats (Sears \& Freedman, 1967). Other field studies (e.g., Barberá et 
al. 2015) observed the tendency to retweet ideologically congenial and uncongenial content. Retweeting a crosscutting post involves multiple distinct processes: seeing the original post (exposure), a desire to share the post, and a motive for sharing the post - a motive that could be a desire to communicate approval, criticism, or even sarcastic mockery. Without much more nuanced coding, it is impossible to know exactly what retweets mean. The degree to which the behaviors observed in these field studies relate to the phenomenon of selective exposure is therefore not very clear. Perhaps the observed heterogeneity in findings with respect to the ideological (a)symmetry question is attributable to the variable sets of contextual factors and psychological phenomena encapsulated in observed behaviors.

Studying selective exposure in naturalistic contexts, such as on social media, captures the phenomenon as it occurs in the real world. Although high in external validity, an accompanying limitation of real world studies is that many factors play a role in manifesting a behavioral trend, complicating the interpretation of the results. Although studies conducted in an artificial setting have limited external validity, a benefit of these studies is that they do offer more control over contextual factors and thus permit a more focused examination of a single psychological element.

Research investigating the ideological (a)symmetry of related social cognitive functions provide reasons to expect that leveling the contextual playing field may reveal ideological symmetry at the basic psychological level. For instance, research finding that conservatives are more prejudiced against minorities typically asked people to offer their opinions about minorities with which liberals tend to sympathize more, such as African Americans (Chambers, Schlenker, \& Collison, 2013). Research supporting the ideological conflict hypothesis studied a variety of groups, including some that liberals are more likely to find threatening than conservatives, such 
as Evangelical Christians, and found that liberals and conservatives are similarly intolerant of groups that challenge their own ideology (Brandt, Reyna, Chambers, Crawford, \& Wetherell, 2014; Crawford, Brandt, Inbar, Chambers, \& Motyl, 2016). Other studies found that, at a basic cognitive/emotional level, and counter to what some previous studies had suggested, conservatives and liberals may be similarly simple-minded (Conway et al., 2015; Gruenfeld, 1995), closed to new experiences (Brandt, Chambers, Crawford, Wetherell, \& Reyna, 2015), prone to sacralize mundane objects (Frimer, Tell, \& Haidt, 2015; Frimer, Motyl, \& Tell, 2017), obedient to authority (Frimer, Gaucher, \& Schaefer, 2014), reverent to moral heroes (Frimer, Biesanz, Walker, \& Mackinlay, 2013), and self-righteous (Waytz, Young, Ginges 2014).

These new findings stop short of suggesting that liberals and conservatives are psychologically equivalent in every respect; a recent meta-analysis suggests otherwise (Onraet et al., 2015). Rather, studies finding ideological symmetry where asymmetries were previously found suggest that research designs that level the contextual playing field are helpful to assess whether liberals and conservatives are psychologically different. To our knowledge, no previous research has systematically tested whether liberals and conservatives are similarly motivated to avoid exposure to crosscutting information on a level contextual playing field. We aim to begin to fill that gap.

\section{The Current Research}

We tested whether liberals and conservatives are similarly or differentially motivated avoid crosscutting information, and whether the same or different underlying psychological processes are at play for both groups. In Study 1, we asked people on both sides of the same-sex marriage debate to either read belief-confirming statements for a chance to win some money or belief-disconfirming statements for a chance to win even more money. The economically 
maximizing choice is to read uncongenial information to win more money. However, if people are motivated to avoid crosscutting information, then people on both sides of the debate should incur the cost of choosing to read belief-confirming statements. We tested whether liberals or conservatives were more likely to forego a chance at more money to remain in their ideological bubbles, or whether they were similarly likely to do so. We also tested whether any possible similarity might be the product of a suppression effect. Namely, if one side of the debate were more ideologically extreme than the other, and if extremists are more prone to selective exposure than moderates, then extremism could suppress an inherent ideological asymmetry.

In Studies 2-3, we introduced a complementary and face valid self-report measure of motivation, and asked people how interested they were in hearing from like- and unlike-minded voters in a past and future election in the U.S. and in Canada. Again, we predicted that both sides would report a greater interest in hearing from their own side than from the other side, and tested whether one side did so to a larger degree. We also tested and aimed to rule out the possibility that prior knowledge explained the motivation to avoid crosscutting information; our prediction was that both sides would similarly demonstrate poorer knowledge of the other side compared to their own side. Study 4 tested whether the motivation to avoid crosscutting information generalizes to several other Culture War issues, such as abortion, gun control, and legalizing marijuana. Study 5 aimed to explain why ideologically minded people avoid crosscutting information by examining whether people anticipate negative personal and interpersonal consequences should they consume crosscutting information. We tested whether liberals and conservatives think that hearing out the other side would induce negative affect (personal) and harm relationships (interpersonal). We conclude with a meta-analysis of the findings from all the studies. In all 5 studies, we tested competing predictions that the motivation underlying selective 
exposure is more common on one side of the political divide, or whether the motivation was symmetrical on the political left and right.

\section{Study 1}

We tested whether liberals or conservatives are more likely to give up a chance to earn $\$ 3$ to get out of having to hear from the other side on the topic of legalizing same-sex marriage.

\section{Method}

Sample. This and all subsequent samples were recruited from Crowdflower, an online crowdsourcing platform similar to Mechanical Turk. Assuming a selective exposure effect size of $d=0.46$ for political issues (Hart et al., 2009), we required $N=152$ for between-subject designs (such as Study 1) and $n=40$ within each cell of within-subject designs (Studies 2-5) to achieve $80 \%$ statistical power. We used this power analysis in this and all subsequent studies. The difference in effect size for liberals and conservatives is unknown, complicating a power analysis. Our approach is to test for any differences within individual studies, then perform a meta-analysis at the conclusion.

In Study 1, we recruited 202 Americans (age 18 or older), of whom 73\% were for samesex marriage and $27 \%$ were against. The sample was 35 years old on average $(S D=13$, range 18-72), $43 \%$ female and $53 \%$ male, $77 \%$ Caucasian, a median household income of $\$ 40,000$ to $\$ 50,000$, and had 5.0 years of post-secondary education $(S D=2.6)$. Data were collected in September 2015, shortly after the U.S. Supreme Court ruled that banning same-sex marriage at the state level was unconstitutional.

Procedure. Participants indicated whether they were for or against same-sex marriage (SSM). The survey then assigned participants to read and answer questions about counterattitudinal statements and to enter a drawing for $\$ 10$. However, participants had the option to 
instead read and answer questions about attitude-confirming statements to enter a drawing for $\$ 7$. Finally, participants reported demographics, which included a measure of ideological extremism.

Opinion about SSM. The question asked, "Do you think that same-sex marriage should be legal everywhere in the U.S.?" Participants answered either "yes" $(n=148)$ or "no" $(n=54)$.

Task choice measure. Participants who indicated that they were for [against] legalizing SSM read the following instructions:

You and the other participants in this study have been entered into a drawing to win a cash prize. Your drawing prize is currently valued at $\$ 10$. The next task is to read 8 statements that argue against [for] legalizing Same-Sex Marriage (SSM for short) and answer a question about each one. Alternatively, you can read 8 statements that argue for [against] legalizing SSM on the condition that your draw prize will be valued at $\$ 7$. Participants then indicated their choice by responding to the following question, "Which set of statements will you read?" with the options being "8 statements arguing in favor of legalizing SSM" or "8 statements arguing against legalizing SSM". They then read and answered questions that matched the set they chose (see the Supplemental Materials for details).

Ideological extremism. The item asked participants to report their political ideology on social issues on a slider scale anchored at -100 (extremely liberal), 0 (moderate), and 100 (extremely conservative). The sample was ideologically diverse, $M=-6, S D=62$. We operationalized ideological extremism as a participant's deviation from zero, calculated as the absolute value of their ideological score $(M=49, S D=36)$.

\section{Results}

If people are motivated to avoid exposure to crosscutting views, they should forsake material interests, such as money, to not hear from the other side. As such, we predicted that 
most people on both sides of the issue would choose the option of reading belief-confirming statements to enter a lottery to win $\$ 7$ more frequently than they would choose to read beliefdisconfirming statements for entering a lottery to win \$10. Consistent with this hypothesis, $63 \%$ of participants (127 of 202) chose to give up a chance at $\$ 3$ to avoid hearing from the other side, a proportion greater than one would expect if participants were selecting randomly between the two options, test for goodness of fit $\chi^{2}(1, N=202)=9.78, p=.002, \varphi=.22$. The two sides had similar proportions of people who chose to read belief-confirming statements- $64 \%$ of pro-SSM people (94 of 148) and 61\% of anti-SSM people (33 of 54)—which did not differ from one another, test for independence $\chi^{2}(1, N=202)=0.10, p=.75, \varphi=.02$.

If one side of the SSM debate were more ideologically extreme than the other, and if ideological extremists are more prone to selective exposure than moderates, then the failure to detect a difference between liberals and conservatives may be the result of a suppression effect. The more moderate side of the debate may be inherently more motivated to avoid crosscutting information than the other. We tested this possibility and found no evidence to support it. The pro-SSM side $(M=48.23, S D=34.93)$ and the anti-SSM side $(M=51.57, S D=37.65)$ were similarly extreme in their ideology, independent samples $t(200)=-0.59, p=.56, d=-0.09$. And ideological extremism did not predict selective exposure. In a logistic regression predicting the decision to read belief-confirming statements, we entered extremism and attitudes toward SSM as predictors. Extremism did not predict selective exposure, Wald's $\chi^{2}=0.83, p=.36$, odds ratio $=1.004,95 \% \mathrm{CI}=[0.996,1.012]$, nor did attitudes toward SSM, Wald's $\chi^{2}=0.73, p=.73$, odds ratio $=1.059,95 \% \mathrm{CI}=[0.768,1.461]$. That is, even when holding extremism constant, both sides of the SSM debate exhibited a similar inclination toward selective exposure. 
We directly replicated the primary effects in a new sample of 245 Americans. Of them, $62 \%$ chose to give up $\$ 3$ to avoid hearing from the other side, a proportion greater than one would expect by chance, $\chi^{2}(1, N=245)=11.94, p<.001, \varphi=.22$. Both sides had similar proportions of participants who engaged in selective exposure- $61 \%$ of pro-SSM people and $63 \%$ of anti-SSM people $-\chi^{2}(1, N=245)=0.08, p=.77, \varphi=.02$.

\section{Discussion}

Faced with the option of entering a drawing to win $\$ 10$ or $\$ 7$, the economically maximizing choice was to compete for $\$ 10$. However, the $\$ 10$ prize required reading a counterattitudinal passage whereas the $\$ 7$ prize allowed the participant to read an attitude-affirming passage. This situation pitted economic self-interest motives (for money) against motives to avoid counter-attitudinal information. Approximately two-thirds of people willingly forfeited a chance at earning an additional $\$ 3$ to get out of having to hear from the other side.

This study had some important limitations. The absence of a control condition, in which the reading options neither affirmed nor conflicted with participants' beliefs, limited the inferences that can be made from the finding that $\sim 60 \%$ of people chose to enter the drawing for \$7. Future research should include such a control condition. Additionally, participants were not explicitly told the odds of winning. This ambiguity limits the interpretation of the finding that $\sim 60 \%$ of people chose to compete for a smaller prize. If liberals and conservatives assumed that the odds were different from one another, this prospective asymmetry could have suppressed a motivational asymmetry. Future research should also explicitly communicate the odds of winning to participants.

To allay concerns about a single study, we sought to replicate this basic finding using other, complimentary methods. Our approach in Studies 2-5 is to use a more direct means of 
inferring motivation - self-reports. In Study 2, we tested whether these effects generalized to other social/political issues, such as voting preference. In addition, we examined whether and aimed to rule out the possibility that prior knowledge may mask an asymmetry in desires for selective exposure. Being or feeling informed may obviate the need for further exposure.

\section{Study 2}

The goal of Study 2 was to test whether liberals or conservatives are more motivated to avoid crosscutting information using a new method and new issue. We asked people who voted for Obama or Romney in the 2012 U.S. Presidential election how interested they were in learning about why people voted the way they did in 2012.

\section{Method}

Sample. Of the 179 Americans who completed the survey, we dropped the 17 who reported to have not voted in 2012 and the 6 who voted for a candidate other than Romney or Obama, leaving a final sample of 156 (48 voted for Romney and 108 for Obama). Power analyses (assuming $d=0.46$ ) indicated that the pro-Romney sample had an $88 \%$ likelihood of detecting the confirmation bias, and the pro-Obama sample had $99.7 \%$ chance. The sample was $59 \%$ female and $41 \%$ male, 38 years old on average $(S D=12)$, and ideologically diverse $(M=-$ 4, $S D=68)$.

Procedure. Participants reported how interested they were in learning more about their ideological allies and opponents and provided demographics, which included an item about how they voted in 2012. We collected this sample in April, 2015. Before completing the motivation measure, we administered a knowledge assessment. The goal was to test and rule out the possibility that desires to avoid learning about the other side were the result of already being knowledgeable. We found no evidence to support this possibility (see the Supplemental 
Materials). Rather, people displayed more knowledge about their ideological allies than they did about their opponents. In a follow-up study, we examined whether people also feel more knowledgeable about their allies than their opponents and again found supportive evidence (see the Supplemental Materials). Taken together, these findings suggest that the motivation to avoid crosscutting information is probably not attributable to already feeling well versed about one's ideological opponents.

Interest. Participants responded to the question, "How interested are you in each of these activities?" on a slide scale anchored at -100 (very uninterested), 0 (neutral), and 100 (very interested). The slider's original position was at 0 (neutral): to indicate an approach motivation participants needed to move the slider to the right, and to indicate an avoidance motivation, they needed to move it to the left. Participants rated nine items, which appeared in randomly intermixed order. The first two items ("hearing from a typical [Obama/Romney] voter about why they voted for [Obama/Romney]" were the main dependent measures. The remaining seven items served to give the scale intuitive anchoring. The items and their mean interest levels were: "getting an electric shock" (-84), "having a tooth pulled" (-78), "ripping a Band-Aid off a burn that is partly healed" (-77), "watching paint dry" (-75), "sitting quietly with your thoughts for 15 minutes" (+39), "going for a walk on a sunny day" $(+64)$, and "re-watching your favorite movie of all time" $(+65)$.

\section{Results}

If people are motivated to avoid crosscutting information, they should be less interested in learning about their opponents' reasons for voting than their allies' reasons. We therefore predicted that there should be an interaction between candidate preference (Romney/Obama) and interest in learning about these candidates. Consistent with this hypothesis, a 2 (judge; between) 
$\times 2$ (target; within) mixed model ANOVA, predicting levels of interest, produced the predicted interaction, $F(1,154)=87.49, p<.001, \omega^{2}=.352$ (along with a marginally significant effect for target, $F(1,154)=3.60, p=.06, \omega^{2}=.011$, and no main effect for judge, $F(1,154)=0.68, p=.41$, $\left.\omega^{2}<.001\right)$.

Figure 1 shows how Obama voters expressed greater interest in hearing about the reasons for why they voted from Obama supporters than from Romney supporters, $F(1,154)=45.18, p$ $<.001, d=0.65$. Similarly, Romney voters expressed greater interest in hearing about the reasons why they voted for Romney supporters than Obama supporters, $F(1,154)=45.71, p$ $<.001, d=-0.95$. We tested whether one side exhibited a greater motivation to avoid crosscutting information than the other by recoding the targets as either ideologically similar or dissimilar and running a 2 (judge: Obama voter, Romney voter) $\times 2$ (target: similar, dissimilar) mixed model ANOVA. A marginally significant interaction, $F(1,154)=3.60, p=.06, \omega^{2}=.011$, suggests that Romney supporters were slightly more motivated to not hear from Obama supporters compared to the vice versa.

Like in Study 1, holding extremism constant between the two sides did not alter the pattern of results. A 2 (judge: Obama voter, Romney voter) $\times 2$ (target: similar, dissimilar) mixed model ANCOVA, with political extremism as a covariate, produced the same judge $\times$ target interaction, $F(1,153)=5.02, p=.03, \omega^{2}=.023$, a marginal effect for target, $F(1,153)=2.79, p$ $=.09, \omega^{2}=.010$, no main effect for judge, $\left.F(1,153)=0.67, p=.41, \omega^{2}<.001\right)$. 
Figure 1. Motivation to avoid crosscutting information in the past, 2012 U.S. Presidential election. Error bars represent $95 \%$ confidence intervals.

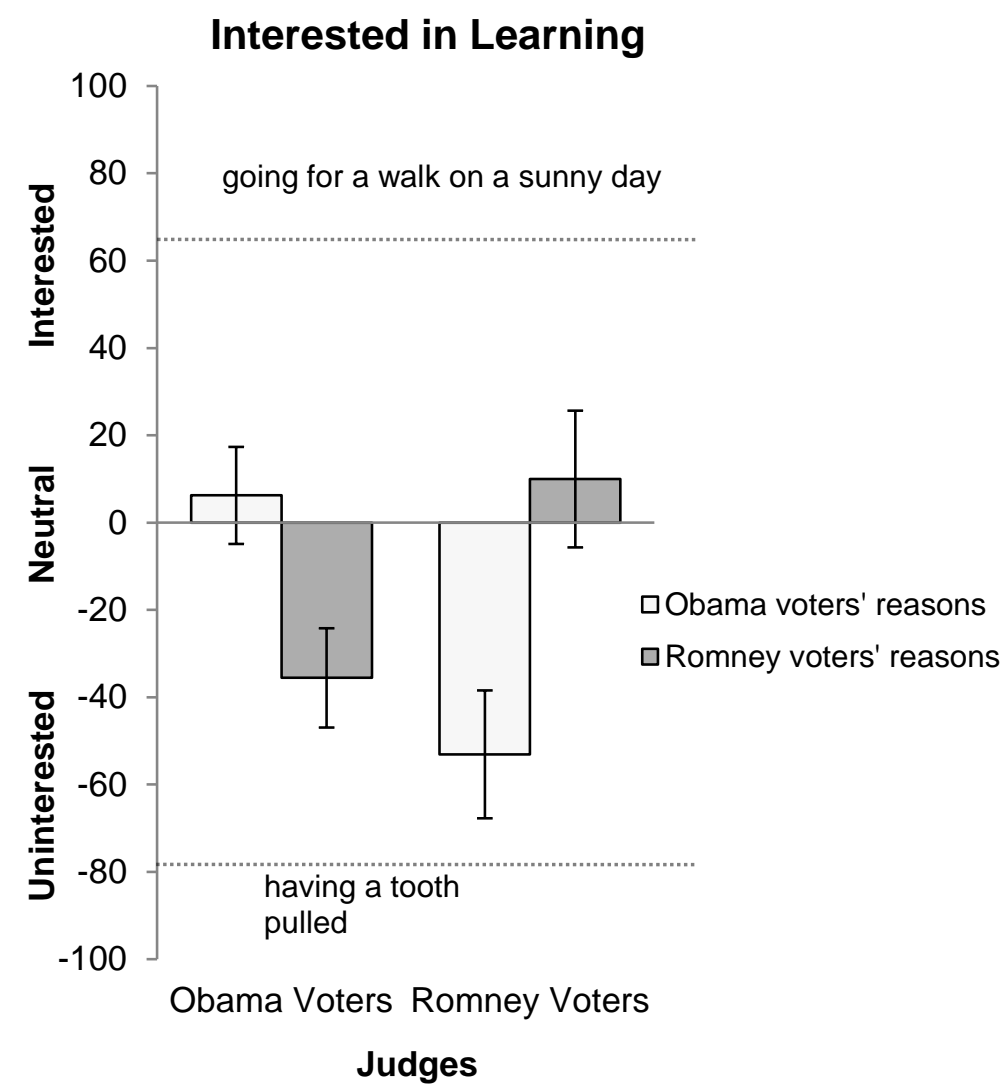

\section{Discussion}

Study 2 provided further evidence of liberals' and conservatives' motivation to avoid crosscutting information. People who voted in the 2012 U.S. Presidential election did not know and did not feel that they knew about their ideological opponents' reasons for their vote (see the Supplemental Materials), nor did they want to know. This desire to avoid crosscutting information applies to both same-sex marriage (Study 1) and to past voting preference in the U.S. (Study 2). Unlike in Study 1, however, we found a marginally significant difference, with conservatives reporting a slightly stronger motivation to avoid crosscutting information than liberals. We revisit this issue in later studies and an eventual meta-analysis.

Why do people desire to avoid learning about their ideological opponents? Perhaps the aversion to political opponents' reasoning in a past election is a product of election fatigue. After 
years of seeing ads, media coverage, and hearing speeches, and finally voting, people may feel ready to move on and think about something else. To rule out this explanation for the results of Study 2, Study 3 examined whether liberals and conservatives report a desire to avoid hearing from one another in the context of a future (and not only a past) election, and also tested hypotheses in two electoral contexts: The U.S. and Canada.

\section{Study 3}

Study 3 tested whether people report an aversion to crosscutting information when thinking about a future national election. We included both upcoming U.S. and Canadian national elections to test whether this aversion to crosscutting information exists in a context that is somewhat less polarized than the U.S. (namely, Canada). At the time of data collection in the U.S., the 2016 Presidential primary campaigning was just about to begin, and Donald Trump (the eventual victor) had not yet launched his campaign. At the time of data collection in Canada, three major political parties were polling almost identically in the upcoming 2015 national election (one month away). The parties were the right wing Conservative Party (leader Stephen Harper; incumbent), the centrist Liberal Party (leader Justin Trudeau; eventually elected), and the left wing New Democrat Party (NDP, leader Thomas Mulcair). In this study, we focused on interest in the more polarized leaders (Harper and Mulcair) because they have a clear ideological leaning (the Liberal Party is politically moderate).

\section{Method}

Sample. In the American sample, we dropped 15 people who did not vote or who voted for a $3^{\text {rd }}$ party candidate in 2012 , leaving $N=145$. The American sample (102 Obama supporters, 99.6\% power, and 43 Romney supporters, $84 \%$ power) was $52 \%$ female and $48 \%$ male, 37 years old on average $(S D=12)$, and slightly liberal on average $(M=-11, S D=62)$. In the Canadian 
sample, we dropped 9 people who were planning on voting for the far-left Green party or the separatist Bloc Québécois. The Canadian sample $(N=146)$ had 28 Conservatives (65\% power), 30 Liberals (center left party; 68\% power), 41 New Democrats (NDP; left wing; 82\% power), and 47 undecided voters, and was $57 \%$ female and $42 \%$ male, 39 years old on average $(S D=14)$, and slightly liberal on average $(M=-7, S D=50)$.

Procedure. Participants reported how interested they were in learning about the candidates' supporters, and provided demographic information, which included an item about their political leaning. In the U.S. sample, the item asked how they voted in 2012 (see Study 2). In Canada, the item asked about voting intentions in the Federal election scheduled for October 19, 2015. We collected the U.S. data in April, 2015 and the Canadian data in September, 2015. To once again test whether prior knowledge explains the desire to avoid crosscutting information, we also asked participants to report how knowledgeable they felt about their allies and opponents. And once again, participants indicated that they felt more knowledgeable about their allies than their opponents (see the Supplemental Materials), further ruling out the possibility that prior knowledge explains the motivation to avoid crosscutting information.

Interest. We used the same interest question as in Study 2, only changing Obama and Romney to the future candidates for the given study (Hillary Clinton and Jeb Bush for the U.S. and Mulcair and Harper for Canada). We also changed the grounding items to be (Ms for US/Canada interest levels in parentheses): "find a \$10 bill” (+68/+68), "take out the trash" (-20/24), "stand in a line-up for 20 minutes" (-48/-51), "read a really boring newspaper article" (-56/51), and "have a tooth pulled" (-64/-66). In the U.S. sample, we also collected data on Marco Rubio (Republican) and Bernie Sanders (Independent; running as a Democrat). Results were generally similar to those for Jeb Bush and Hillary Clinton, respectively. Romney voters were 
more interested in hearing from Rubio supporters $(M=+6)$ than from Sanders' supporters $(M=$ $42), t(42)=4.39, p<.001, d=0.67$. And Obama voters were more interested in hearing from Sanders supporters $(M=-6)$ than from Rubio supporters $(M=-25), t(101)=3.53, p<.001, d=$ 0.35 .

Political extremism. We used the same measure as in Studies 1 and 2.

\section{Results}

2016 U.S. Presidential election. We predicted an interaction between candidate preference and interest in learning about candidates. Consistent with the hypothesis, a 2(judge; Obama voter, Romney voter; between $) \times 2($ target; Clinton, Bush; within $)$ mixed model ANOVA yielded an interaction, $F(1,143)=43.55, p<.001, \omega^{2}=.227$ (along with a main effect of judge, $F(1,143)=4.75, p=.03, \omega^{2}=.025$, and no main effect of target, $F(1,143)=0.08, p=.78, \omega^{2}$ $<.001)$. Obama voters expressed greater interest in hearing from Clinton supporters than from Bush supporters, $F(1,143)=39.96, p<.001, d=0.77$, and Romney supporters displayed the opposite pattern, $F(1,143)=14.17, p<.001, d=-0.74$ (see Figure 2). Controlling for extremism did not alter the pattern of results. A 2(judge; Obama voter, Romney voter; between) $\times 2$ (target; Clinton, Bush; within) mixed model ANCOVA with extremism as a covariate still yielded a judge $\times$ target interaction, $F(1,142)=42.84, p<.001, \omega^{2}=.208$.

We tested whether one side expressed greater interest in avoiding crosscutting information than the other by recoding the targets as either ideologically similar or dissimilar and running a 2 (judge: Obama voter, Romney voter) $\times 2$ (target: similar, dissimilar) mixed model ANOVA. The interaction effect was not significant, $F(1,143)=0.08, p=.78, \omega^{2}<.001$ (even when controlling for extremism in an ANCOVA, $\left.F(1,142)<0.01, p=.97, \omega^{2}<.001\right)$, meaning 
that liberals and conservatives exhibited similarly intense desires to remain in their respective information bubbles.

Figure 2. Interest levels in learning about why people planned on voting the way they did in the 2016 U.S. Presidential election (Study 3). Error bars represent 95\% confidence intervals.

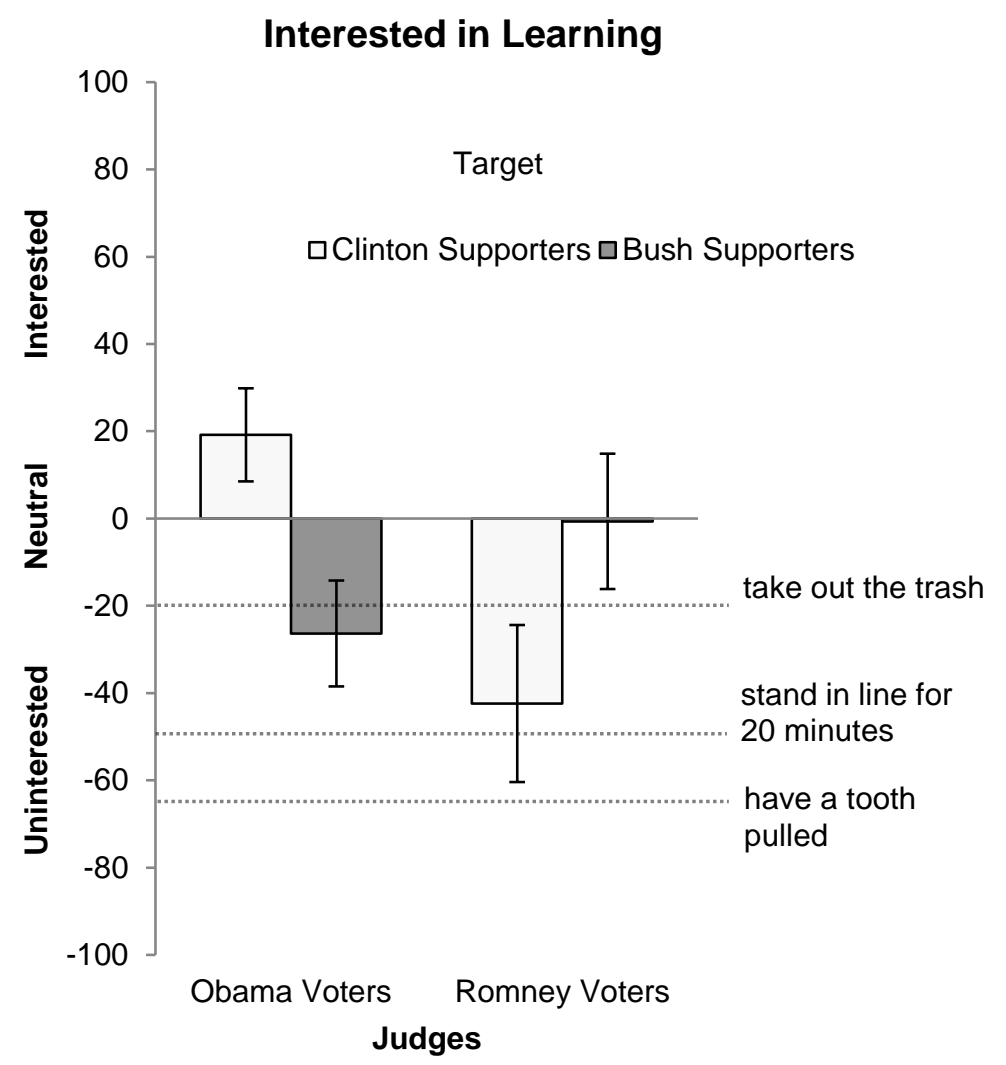

To give the intensity of the motivation to avoid crosscutting information heuristic meaning, we compared interest in hearing from the other side and a variety of aversive tasks (using a Bonferroni correction for 8 contrasts). Partisans were slightly more interested in taking out the trash than hearing from one another (albeit, non-significantly so; see Table 1). Relative to hearing out the other side, both sides expressed non-significantly, but slightly more aversion toward standing in line for 20 minutes. And both sides indicated that they would prefer to hear from the other side over having a tooth pulled. 
Table 1. Participants' reported interest in hearing from the other side compared to a set of comparison tasks. Statistics represent within-subject planned contrasts, such that positive effects mean that people would rather hear from the other side than perform this task, and vice versa for negative effects (U.S. sample, Study 3).

\begin{tabular}{llllll}
\hline Comparison Task & \multicolumn{2}{c}{ Obama Voters } & & \multicolumn{2}{c}{ Romney voters } \\
\cline { 2 - 3 } \cline { 5 - 6 } & \multicolumn{1}{c}{$(\mathbf{1 0 1})$} & $\boldsymbol{d}$ & & $\boldsymbol{t}(\mathbf{4 2})$ & $\boldsymbol{d}$ \\
\hline Take out the trash & -1.43 & -0.14 & & -1.45 & -0.22 \\
Stand in a line-up for 20 minutes & +2.32 & +0.23 & & +1.01 & +0.15 \\
Read a really boring newspaper article & $+3.60^{* * *}$ & +0.36 & & +1.95 & +0.30 \\
Have a tooth pulled & $+4.34 * * *$ & +0.43 & & $+2.97 *$ & +0.45 \\
\hline
\end{tabular}

Note. Bonferroni corrected ${ }^{\dagger} p<.0125, * p<.00625, * * p<.00125, * * * p<.000125$

2015 Canada Federal Election. We similarly predicted an interaction between voting intention and interest in learning about like- versus unlike-minded voters' views in the Canadian context. Consistent with the hypothesis, a 4(judge: Conservative, Liberal, NDP, Undecided; between $) \times 2$ (target: Harper, Mulcair; within) mixed model ANOVA yielded an interaction, $F(3,142)=27.30, p<.001, \omega^{2}=.326$ (along with main effects of judge and target, $F \mathrm{~s} \geq 4.87, p$ $\left.\leq .003, \omega^{2} \geq .066\right)$, as did a 2(judge: Conservative, NDP) $\times 2($ target: Harper, Mulcair) mixed model ANOVA, $F(1,67)=72.07, p<.001, \omega^{2}=.486($ and a main effect of target $, F=6.25, p$ $=.02, \omega^{2}=.036$, but no main effect of judge, $\left.F=2.38, p=.13, \omega^{2}=.020\right)$.

Figure 3 shows how leftist partisans (Liberals and NDP) expressed greater interest in hearing from Mulcair-supporters than from Harper-supporters, $F \mathrm{~s}(1,142) \geq 13.61, p \mathrm{~s}<.001, d \mathrm{~s} \geq$ 0.61, and vice versa for Conservative partisans, $F(1,142)=15.60, p<.001, d=-0.86$. Undecided voters' did not differ in their interest in learning about the reasons why supporters would vote for the left/right candidates, $F(1,142)=0.44, p=.51, d=0.10$. We tested whether people on the left (NDP) or on the right (Conservative) exhibited greater interest in remaining in their ideological bubble than the other by recoding the targets as either ideologically similar or dissimilar and running a 2 (judge: Conservative, NDP) $\times 2$ (target: Harper, Mulcair) mixed model ANOVA. A 
significant interaction, $F(1,67)=6.25, p=.02, \omega^{2}=.036$ (largely unchanged by controlling for extremism in an ANCOVA, $\left.F(1,66)=4.67, p=.03, \omega^{2}=.043\right)$, suggests that people on the left, $F(1,142)=76.91, p<.001, d=1.50$, reported a greater interest in avoiding crosscutting information than people on the right, $F(1,142)=15.60, p<.001, d=0.86$.

Figure 3. Interest levels in learning about why people plan on voting the way they were planning to in the upcoming 2015 Canadian Federal election (Study 3). Parties include the Left Wing NDP, Center-Left Liberal Party, and Right Wing Conservative Party.

\section{Interested in Learning}

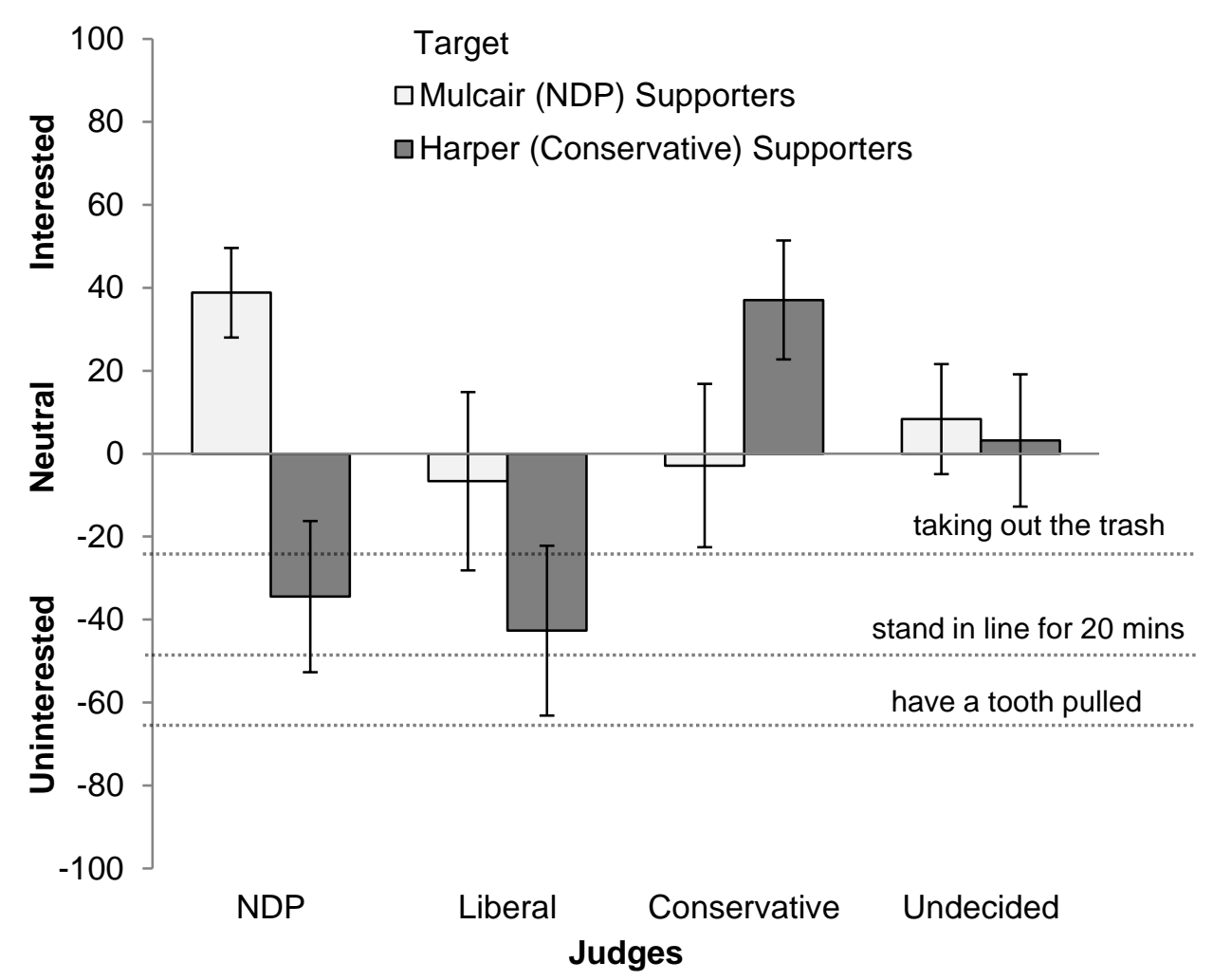

For heuristic value, we next compared participants' interest in hearing from the other side compared to various comparison tasks using one-sample planned contrasts and a Bonferroni correction for 8 contrasts. NDP voters' interest in hearing from Harper supporters was slightly 
colder than their interest in taking out the trash, and only slightly warmer than standing in line for 20 minutes, reading a really boring news article, and having a tooth pulled (see Table 2). Harper supporters exhibited a relatively similar pattern.

Table 2. Participants' reported interest in hearing from the other side compared to a set of comparison tasks. Statistics represent within-subject planned contrasts, such that positive effects mean that people would rather hear from the other side than perform this task, and vice versa for negative effects (Canada sample, Study 3).

\begin{tabular}{lccccc}
\hline \multirow{2}{*}{ Comparison Task } & \multicolumn{2}{c}{ NDP Voters } & & \multicolumn{2}{c}{ Conservative voters } \\
\cline { 2 - 3 } \cline { 5 - 6 } & \multicolumn{1}{c}{$(\mathbf{4 0})$} & $\boldsymbol{d}$ & & $\boldsymbol{t}(\mathbf{2 7})$ & $\boldsymbol{d}$ \\
\hline Take out the trash & -0.48 & -0.08 & & +0.42 & +0.08 \\
Stand in a line-up for 20 minutes & +1.85 & +0.29 & & +2.66 & +0.50 \\
Read a really boring newspaper article & +2.52 & +0.39 & & $+2.90^{\dagger}$ & +0.55 \\
Have a tooth pulled & $+3.70^{* *}$ & +0.58 & & $+3.26^{*}$ & +0.62 \\
\hline
\end{tabular}

Note. Bonferroni corrected ${ }^{\dagger} p<.0125, * p<.00625, * * p<.00125$

Next, we used undecided voters as a control group to test whether Canadians sought to confirm their beliefs and, independently, avoid disconfirming information. Compared to undecided voters, NDP voters were more interested in learning about other NDP voters, $t(86)=$ 3.43, $p=.001, d=0.74$, and less interested in learning about Conservative voters, $t(86)=-3.06$, $p=.003, d=-0.65$. Compared to undecided voters, Conservative voters were more interested in learning about other Conservative voters, $t(73)=2.83, p=.006, d=0.71$, but no less interested in learning about NDP voters, $t(73)=-0.96, p=.34, d=-0.22$. (Making a Bonferroni correction for the 4 contrasts reduces the critical $\alpha=.0125$, leaving the pattern of results unchanged.) In summary, participants were consistently motivated to confirm their beliefs. Moreover, leftist NDP voters (but not rightist Conservatives) were also motivated to avoid belief disconfirmation. 


\section{Discussion}

Study 3 again found evidence of desires to avoid crosscutting information, both for people on the left and on the right, but now in the context of a future rather than a past election, and in the US and Canada. Studies 1-3 established the prevalence of desires to avoid exposure to the ideas of unlike-minded people, and ruled out the possibilities of prior knowledge and issue fatigue as alternative explanations for why people are uninterested in learning more about their ideological opponents' reasoning.

\section{Study 4}

In Study 4a, we tested whether belief confirmation motives are at play and (a)symmetric in a variety of other contentious issues in the US. The aim of Study 4 was to test whether the effects concerning SSM (Study 1) and voting preference (Studies 2 and 3) generalize to other Culture War issues.

\section{Method}

Sample. We recruited a sample of Americans $(N=190)$. The sample was $47 \%$ female and 52\% male, 32 years old on average $(S D=13)$, and somewhat liberal on average $(M=-14$, $S D=56)$. See Table 4 for power analyses.

Procedure. For each issue, participants reported their opinions and then indicated how interested they were in hearing from like- and unlike-minded others. There were seven issues in total, which appeared in counterbalanced order between participants. Data were collected in May 2016, at which time Donald Trump was the presumptive nominee for the Republican Party in the upcoming 2016 U.S. Presidential election. Within the Democratic Party, both Hillary Clinton and Bernie Sanders remained contenders. 
Opinions. We used the same type of question as in Study 2, only adjusting the content to the various issues. The issues were: (a) whether marijuana should be legal everywhere in the U.S., (b) whether climate change is real and human-caused, (c) whether the federal government should impose restrictions on the sales of firearms, (d) whether participants identify as pro-life or pro-choice on the abortion issue, (e) whether Donald Trump would make a good President, (f) whether Bernie Sanders would make a good President, and (g) whether Hillary Clinton would make a good President. Participants indicated whether they were for one side or the other, or had no preference. We included in our analyses only responses that took a side. The social political ideologies of the two sides differed in the predicted direction on all seven issues, with all $p s<.03$ and an average $d=0.73$ (minimum $d=0.36$ for Marijuana, maximum $d=0.92$ for abortion $\&$ climate change).

Interest. Immediately after indicating their opinion, participants reported how much interest they had in hearing from people on each side of the issue tell them about their opinion. The measure was analogous to that used in Studies 2 and 3.

Political extremism. We used the same measure as in previous studies.

\section{Results}

If people are motivated to avoid hearing from their ideological opponents, we should see an interaction between the judge's opinion and the target's opinion in predicting interest. Consistent with this hypothesis, a 2(judge; for, against; between) $\times 2$ (target; for, against; within) mixed model ANOVA yielded an interaction for all seven issues (see Table 3). That is, Americans consistently reported greater interest in hearing from like- versus unlike-minded others. We also found a theoretically tangential main effect for judge on five of seven issues. For example, Donald Trump supporters reported greater interest overall than Trump opponents. 
Examining the simple main effects for each side of each issue (see Table 4), we found a preference for congenial over uncongenial views in 13 of 14 cases. The group that exhibited the most motivation to avoid crosscutting information was Donald Trump supporters. The one group that did not significantly exhibit the effect was climate change deniers. However, the small sample of deniers (and low power; see Table 4) may mean that the sample size prevented us from detecting a real effect.

Recoding the targets as either congenial (like-minded) or uncongenial (unlike-minded) and re-running the ANOVA allowed us to test whether desires to remain within one's ideological bubble is ideologically asymmetric. We consistently found null interactions, meaning that we found no evidence of asymmetry. The one exception, which yielded just-significant results, was climate change (believers > deniers). Controlling for ideological extremism in an ANCOVA yielded similar results (see Table 3 ).

Given the nested nature of the data (seven reported interest levels nested within participants), multilevel modeling would provide a statistically powerful means of detecting a difference between liberals and conservatives in their preference for congenial over uncongenial views, should one exist. We tested this (see the Supplemental Materials) and, in spite of the enhanced statistical power associated with multilevel analyses, still failed to find a significant difference between liberals and conservatives in their desire to avoid crosscutting information. 
Table 3. Desires to remain in one's ideological bubble on a variety of Culture War issues. Analyses were 2 (judge: for, against) $\times 2$ (target: for, against) mixed-model ANOVAs, predicting interest levels. They consistently produced significant interactions, indicating widespread desires to avoid crosscutting information on the issue. Recoding the target as congenial vs. uncongenial and re-running the ANOVA routinely yielded a null interaction, meaning that desire to remain in an ideological bubble was ideologically symmetric. Controlling for (possible) group differences in ideological extremism in an ANCOVA did little to change the result.

\begin{tabular}{|c|c|c|c|c|c|c|c|c|c|c|}
\hline & \multirow{2}{*}{\multicolumn{6}{|c|}{$\begin{array}{c}\text { For Versus Against } \\
\text { Mixed Model ANOVA }\end{array}$}} & \multicolumn{4}{|c|}{ Asymmetry Test } \\
\hline & & & & & & & \multirow{2}{*}{\multicolumn{2}{|c|}{$\frac{\text { ANOVA }}{\text { Judge } \times \text { Target }}$}} & \multirow{2}{*}{\multicolumn{2}{|c|}{$\frac{\text { ANCOVA }}{\text { Judge } \times \text { Target }}$}} \\
\hline & \multicolumn{2}{|c|}{ Judge } & \multicolumn{2}{|c|}{ Target } & \multicolumn{2}{|c|}{ Judge $\times$ Target } & & & & \\
\hline & $F$ & $\omega^{2}$ & $F$ & $\omega^{2}$ & $F$ & $\omega^{2}$ & $F$ & $\omega^{2}$ & $F$ & $\omega^{2}$ \\
\hline Legalizing Marijuana & $6.29 *$ & .034 & 1.10 & .001 & $24.83 * * *$ & .137 & 1.10 & .001 & 1.11 & .001 \\
\hline Climate Change & $8.37 * *$ & .043 & $4.91 *$ & .021 & $18.65 * * *$ & .095 & 4.91* & .021 & $3.87 *$ & .017 \\
\hline Gun Restrictions & 0.06 & $<.001$ & 0.27 & $<.001$ & $18.21 * * *$ & .113 & 0.27 & $<.001$ & 0.23 & $<.001$ \\
\hline Abortion & $5.89 *$ & .031 & 0.09 & $<.001$ & $29.31 * * *$ & .155 & 0.09 & $<.001$ & 0.05 & $<.001$ \\
\hline Donald Trump as President & $26.43 * * *$ & .140 & 0.07 & $<.001$ & $42.32 * * *$ & .209 & 0.07 & $<.001$ & 0.18 & $<.001$ \\
\hline Bernie Sanders as President & $16.79 * * *$ & .116 & 0.93 & $<.001$ & $19.60 * * *$ & .133 & 0.93 & $<.001$ & 0.66 & $<.001$ \\
\hline Hillary Clinton as President & 2.38 & .009 & 0.13 & $<.001$ & $42.28 * * *$ & .223 & 0.13 & $<.001$ & 0.10 & $<.001$ \\
\hline
\end{tabular}

$* p<.05, * * p<.01, * * * p<.001$ 
Table 4. Participants' reported interest in hearing from like- and unlike-minded others on a variety of Culture War issues. The congenial versus uncongenial contrast statistics represent within-subject planned contrasts, such that positive effects mean that people would rather hear from like- over unlike-minded others. Power analyses assume an estimated effect size of $d=$ 0.46. Bolded numbers are statistically significant.

\begin{tabular}{|c|c|c|c|c|c|c|c|}
\hline \multirow[t]{2}{*}{ Issue } & \multirow[t]{2}{*}{ Judge's Opinion } & \multirow[b]{2}{*}{$n$} & \multirow[b]{2}{*}{ Power } & \multicolumn{2}{|c|}{$\begin{array}{c}\text { Interest in Hearing from Target who } \\
\text { is...M(SD) } \\
\end{array}$} & \multicolumn{2}{|c|}{$\begin{array}{l}\text { Like vs. Unlike } \\
\text { Planned Contrast } \\
\end{array}$} \\
\hline & & & & Liberal & Conservative & $F$ & $d$ \\
\hline \multicolumn{4}{|c|}{ Legalizing Marijuana } & Pro-Legalization & Anti-Legalization & & \\
\hline & Pro-Legalization & 82 & $98 \%$ & $35(59)$ & $-3(67)$ & $20.22 * * *$ & 0.45 \\
\hline & Anti-Legalization & 67 & $96 \%$ & $-17(66)$ & $8(58)$ & $\mathbf{7 . 0 3} * *$ & 0.38 \\
\hline \multicolumn{4}{|c|}{ Climate Change } & a Believer & a Denier & & \\
\hline & Believer & 134 & $>99 \%$ & $43(45)$ & $-3(63)$ & $60.00 * * *$ & 0.65 \\
\hline & Denier & 29 & $67 \%$ & $-14(65)$ & $1(62)$ & 1.34 & 0.24 \\
\hline \multicolumn{4}{|c|}{ Gun Restrictions } & Pro-Restrictions & Anti-Restrictions & & \\
\hline & Pro-Restrictions & 93 & $99 \%$ & $27(51)$ & $-2(59)$ & $18.43 * * *$ & 0.48 \\
\hline & Anti-Restrictions & 42 & $83 \%$ & $-1(60)$ & $21(57)$ & $5.09 *$ & $\mathbf{0 . 3 0}$ \\
\hline \multicolumn{4}{|c|}{ Abortion } & Pro-choice & Pro-life & & \\
\hline & Pro-choice & 83 & $99 \%$ & $8(57)$ & $-21(64)$ & $14.21 * * *$ & 0.42 \\
\hline & Pro-life & 71 & $97 \%$ & $-3(65)$ & $29(60)$ & $15.11 * * *$ & 0.45 \\
\hline \multicolumn{4}{|c|}{ Donald Trump as President } & Anti-Trump & Pro-Trump & & \\
\hline & Anti-Trump & 121 & $>99 \%$ & $21(63)$ & $-29(63)$ & $51.19 * * *$ & 0.62 \\
\hline & Pro-Trump & 35 & $75 \%$ & $20(64)$ & $65(33)$ & $12.52 * *$ & 0.77 \\
\hline \multicolumn{4}{|c|}{ Bernie Sanders as President } & Pro-Sanders & Anti-Sanders & & \\
\hline & Pro-Sanders & 63 & $95 \%$ & $35(53)$ & $14(58)$ & $6.30 *$ & 0.30 \\
\hline & Anti-Sanders & 57 & $93 \%$ & $-28(62)$ & $5(64)$ & $13.85 * * *$ & $\mathbf{0 . 5 3}$ \\
\hline \multicolumn{4}{|c|}{ Hillary Clinton as President } & Pro-Clinton & Anti-Clinton & & \\
\hline & Pro-Clinton & 54 & $91 \%$ & $33(56)$ & $-9(65)$ & $18.80 * * *$ & 0.55 \\
\hline & Anti-Clinton & 90 & $99 \%$ & $-20(64)$ & $18(62)$ & $25.21 * * *$ & 0.58 \\
\hline
\end{tabular}

$* p<.05, * * p<.01, * * * p<.001$

\section{Discussion}

Study $4 \mathrm{a}$ found that the greater interest in congenial versus uncongenial information is common across several Culture War issues. Moreover, we found very little evidence of asymmetry between the two sides of each issue, even when controlling for any possible asymmetries in ideological extremism. 
One limitation of Study 4a was the absence of non-political issues. To test whether liberals or conservatives have a stronger desire to avoid uncongenial information on non-political issues, we conducted a follow-up study (Study $4 \mathrm{~b}, N=177$ ) using the same methods (see the Supplemental Materials for complete details). We again found belief confirmation desires for ideological issues such as same-sex marriage, political party preference, and whether the police should have more freedom or more restrictions. And we also found greater desire to hear from like- versus unlike-mined others on questions such as preferred beverages (Coke vs. Pepsi), seasons (spring vs. autumn), airplane seat (aisle vs. window), and sports leagues (the NFL vs. the NBA). Our main question was whether liberals or conservatives showed a stronger preference for congenial over uncongenial information on non-political topics. And we found little evidence of differences. For both political and non-political information, liberals and conservatives exhibited a similarly strong desire to hear congenial views and avoid uncongenial ones (see the Supplemental Materials). This result suggests that the ideological symmetry in the desire to avoid uncongenial information may not be limited to political topics.

Studies 1-4a and $4 \mathrm{~b}$ established the existence and pervasiveness of desires to favor congenial over uncongenial information, and ruled out the possibilities of prior knowledge and issue fatigue as alternative explanations for why people are uninterested in learning more about their ideological opponents' reasoning.

\section{Study 5}

Why are people on the left and right motivated to avoid disconfirming information? We suggest that two processes may be at play. First, exposure to belief-disconfirming information could create cognitive dissonance and resulting negative affect. And second, hearing beliefchallenging information could undermine a sense of shared reality with close others, thus 
threatening a fundamental need. Using a mediation approach, we tested whether both sides anticipate that listening to belief-disconfirming information will create cognitive dissonance (feelings of threaten, frustration, anger, and the need to exert a lot of effort), and undermine a shared reality with the person expressing discordant views (causing an argument and harming the relationship). In Study 5, we tested whether anticipated intrapersonal and interpersonal negativity mediates the desire to remain in one's ideological bubble, and whether the explanations are the same for liberals and conservatives.

\section{Method}

Participants. We recruited 236 Americans, of which $69 \%$ reported being for the legalization of same-sex marriage ( $>99 \%$ power assuming $d=0.46)$ and the remainder against (97\% power). The sample was $46 \%$ female and $52 \%$ male, and 35 years old on average $(S D=$ 12).

Procedure. Participants indicated whether they were for or against the legalization of SSM (see Study 1), and then were randomly assigned to indicate how interested they were in hearing from someone who does or does not support the legalization of SSM (randomly assigned between subjects), and offer explanations why ${ }^{1}$. The study was a 2 (judge: for, against) $\times 2$ (target: for, against) between subjects design. As ideological extremist did not alter the pattern of findings in previous studies, we dropped the measure in this study.

Interest. The question asked, "How interested are you in hearing someone tell you all about why he/she believes that same-sex marriage should [not] be legal everywhere in the U.S.?"

\footnotetext{
${ }^{1}$ We also included an attention check, which was successful. The check asked participants to agree or disagree with two items: "Hearing from this person would... involve listening to what they say," and "... mean closing my eyes and plugging my ears." Participants agreed more with the former $(M=65, S D=25)$ than with the latter statement $(M=32, S D=29), t(227)=11.88, p<.001, d=0.79$.
} 
Participants responded on a scale anchored at -100 (very uninterested), 0 (neutral), and 100 (very interested).

Explanations (mediators). Immediately following the interest question, the explanation question asked, "Why do you feel that way? Hearing from this person would..." Participants responded on a 101-point scale anchored at 0 (not at all), 50 (somewhat), and 100 (totally). The items were of two types ${ }^{2}$, with reverse scored items indicated by an (R):

- Cognitive dissonance $(\alpha=.68)$ : "cause me to feel angry," "require a lot of effort on my part," "make me feel relaxed" (R), and "make me feel happy" (R)

- Undermined shared reality $(\alpha=.72)$ : "likely result in a fight," "harm my relationship with the speaker," "strengthen mutual respect between me and the speaker" (R), and "build trust between the speaker and me" (R).

A factor analysis of the eight items yielded a two-factor solution. The two factors grouped the positively and negatively worded items (see Table S9 in the Supplemental Materials). Because of our theoretical interest in personal and interpersonal reasons for avoiding crosscutting information, we chose to retain the original scales, and interpret our findings cautiously.

\section{Results}

We begin by testing whether the desire to avoid crosscutting information effect conceptually replicates in a between-subjects design, and found that it does. A 2 (judge) $\times 2$ (target) between-subjects ANOVA, predicting levels of interest, produced the predicted interaction, $F(1,232)=52.66, p<.001, \omega^{2}=.175$, and no main effects, $F \mathrm{~s}(1,232) \leq 0.34, p \mathrm{~s}$

\footnotetext{
${ }^{2}$ We also included and later dropped four additional items tapping the sense that hearing from the other side would display disloyalty to people who share an ideology. The items did not aggregate well $(\alpha=.38)$ and were deemed during the review process to be theoretically tangential to the focus of this paper. The items were: "make me look like I doubt my beliefs," "be unfaithful to the cause that I believe in," "show my friends that I am trustworthy" (R), and "send the right message" (R).
} 
$\geq .56, \omega^{2} \mathrm{~s} \leq .001$. Pro-SSM participants were more interested in reading pro-SSM arguments than anti-SSM arguments, $F(1,235)=38.19, p<.001, d=1.04$, and vice versa for anti-SSM participants, $F(1,235)=21.29, p<.001, d=-0.96$ (see Figure 4$)$. We tested whether people for or against SSM exhibited greater desires to avoid hearing from the other by recoding the targets as either ideologically similar or dissimilar and running a 2 (judge: for, against) $\times 2$ (target: similar, dissimilar) between subjects ANOVA. The interaction was not significant, $F(1,232)=$ $0.20, p=.66, \omega^{2}<.001$, meaning that people on both side of the SSM debate expressed similar levels of desire to remain in their ideological bubbles.

Figure 4. Desires to remain in one's ideological bubble on the topic of legalizing same-sex marriage (SSM). Error bars represent $95 \%$ confidence intervals.

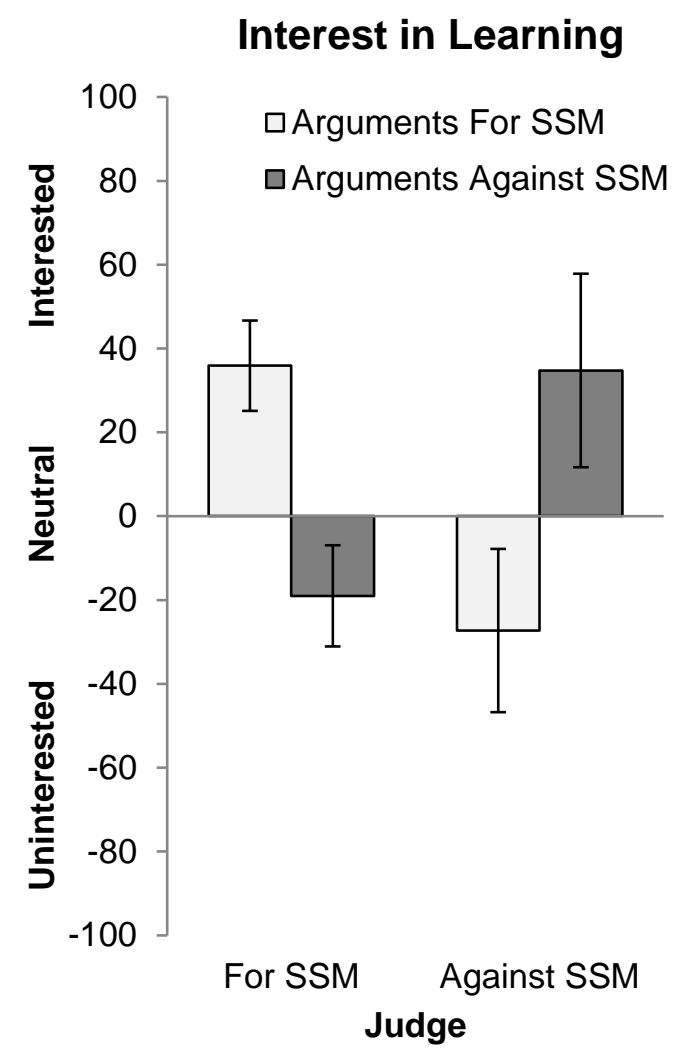

Next, we tested whether anticipated reactions mediated the lower interest in hearing from the other side versus the same side, and tested whether these processes were different for people 
that were for and against SSM. First, we formed two groups: (a) belief-disconfirming (e.g., proSSM people assigned to answer questions about someone who is anti-SSM), and (b) beliefconfirming participants. Next, we ran a bootstrapped moderated mediation analysis (PROCESS models 8 and 15), with group (X) predicting interest level (Y), and the two explanations entered as simultaneous mediators $\left(\mathrm{M}_{i}\right.$; see Figure 5). Ideology (pro- or anti-SSM) was entered as a possible moderator of the $\mathrm{X} \rightarrow \mathrm{M}$ relationship and of the $\mathrm{M} \rightarrow$ Y relationship.

Figure 5. Mediation models testing whether people report desires to remain in their ideological bubble because they anticipate cognitive dissonance and a threat to their shared reality with the other person. We tested whether the same or different process was in effect for people who were for and against same-sex marriage using a moderated mediation model.

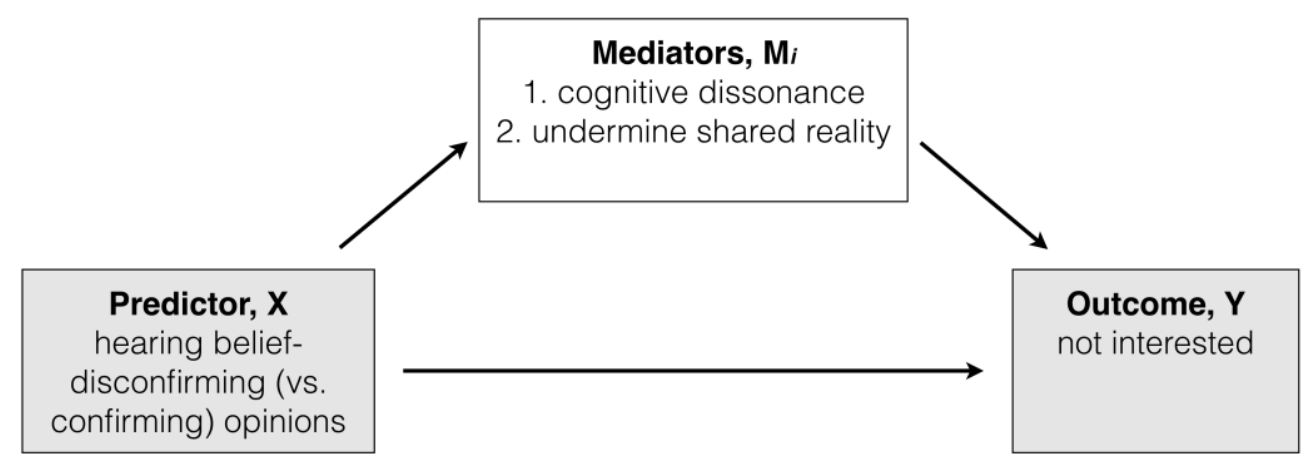

We found that people avoided disconfirming information because they thought it would create cognitive dissonance and undermine a shared reality with the speaker (see Table 5). These processes were the same for people who were for and against SSM, as evidenced by moderated mediation effects that consistently overlapped with zero. We found one exception to these equivalences: anticipated threat to a shared reality predicted an aversion toward hearing beliefdiscordant views, but only for people who were against SSM. 
Table 5. Liberals and conservatives similarly desired to avoid belief-disconfirming opinions on the topic of same-sex marriage (SSM) because of anticipated cognitive dissonance and threats to a shared reality with the other person. Numbers represent unstandardized coefficients [95\% confidence intervals, CIs] of a bootstrapped moderated mediation analysis with the two mediators analyzed simultaneously. Bolded numbers are significant.

\begin{tabular}{|c|c|c|c|c|c|}
\hline \multirow[t]{2}{*}{ Mediators } & \multicolumn{2}{|c|}{ Conditional Direct Effects } & \multicolumn{2}{|c|}{ Indirect Effects } & \multirow{2}{*}{$\begin{array}{l}\text { Moderated } \\
\text { Mediation }\end{array}$} \\
\hline & Anti-SSM & Pro-SSM & Anti-SSM & Pro-SSM & \\
\hline$X \rightarrow M$ & $43.2[21.5,64.8]$ & $19.9[4.4,35.3]$ & & & \\
\hline Cognitive Dissonance & & & $12.7[2.5,28.9]$ & $24.3[13.0,39.5]$ & $11.6[-0.7,28.2]$ \\
\hline Undermine Shared Reality & & & $5.8[0.1,18.3]$ & $9.9[1.2,21.5]$ & $4.1[-0.9,15.3]$ \\
\hline $\mathrm{M} \rightarrow \mathrm{Y}$ & $37.8[15.6,59.8]$ & $22.3[6.6,38.0]$ & & & \\
\hline Cognitive Dissonance & & & $21.7[7.8,38.3]$ & $27.3[14.0,45.1]$ & $5.7[-12.3,26.2]$ \\
\hline Undermine Shared Reality & & & $16.8[5.4 .33 .4]$ & $0.7[-11.0,11.8]$ & $-16.0[-36.1,-0.6]$ \\
\hline
\end{tabular}

\section{Discussion}

Study 5 again replicated the desire to avoid hearing from ideological opponents with people who are for or against the legalization of SSM. Participants then explained that they were uninterested in hearing from the other side because doing so would cause them to experience cognitive dissonance and might harm the relationship with the other person, undermining a sense of a shared reality. Caution is due in interpreting the differences between the personal and interpersonal mediators: a factor analysis did not confirm their distinctiveness.

\section{Summary Analysis}

We summarily tested whether liberals and conservatives are similarly prone to prefer belief-confirming over belief-disconfirming information by meta-analyzing the data from all five studies (See Table S10 in the Supplemental Materials for the data included). We converted group difference statistics ( $\chi^{2}$ or $d$, depending on the study) to equivalent correlation coefficients, $r_{e q}$. Then, using a Hedges-Vevea (1998) random effects model, we found that liberals $(N=1612 ; d=$ $\left.0.62 ; r_{\mathrm{eq}}=.30 ; z=11.36, p<.001\right)$ and conservatives $\left(N=848 ; d=0.58 ; r_{\mathrm{eq}}=.28 ; z=8.11, p\right.$ 
$<.001)$ exhibited similarly strong desires to remain in their ideological bubbles, with their $95 \%$ confidence intervals being virtually identical and almost completely overlapping (see Figure 6).

Figure 6. Meta-analysis of desires to consume congenial over uncongenial information for liberals and conservatives. Small grey dots represent results from individual tests. Large black dots represent meta-analytic results, with error bars being $95 \%$ confidence intervals.

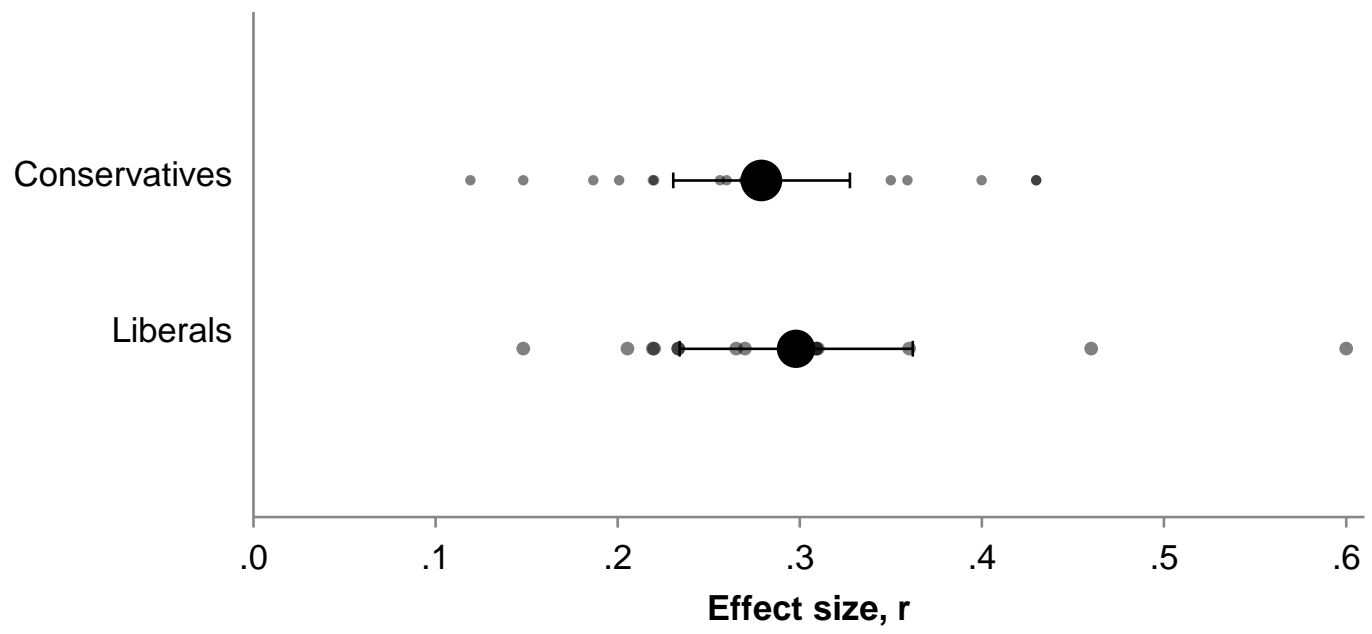

We then investigated whether political ideology (liberal or conservative) moderated the motivation to remain within one's ideological bubble. Of critical importance, ideology did not moderate the effect, $\chi^{2}(1, N=2460)=0.27, p=.61, \varphi=.005$, meaning that liberals and conservatives were similarly motivated to avoid hearing from their ideological opponents. One extreme study result for liberals $(r=.60)$ is nearly a statistical outlier $(z=2.81)$. Eliminating this observation leaves the results unchanged: ideology still did not moderate the effect, $\chi^{2}(1, N=$ 2419) $=0.09, p=.77, \varphi=.002$.

\section{General Discussion}

Five studies found that liberals and conservatives were similarly motivated to avoid hearing one another's opinions on a variety of social issues, and largely for the same reasons. In Study 1, most people on both sides of the same-sex marriage debate willingly passed up on a 
chance to win an extra $\$ 3$ to avoid having to hear from the other side. And Studies 2-4 found that people on both sides of recent and upcoming national elections in the US and Canada, as well as both sides of an array of Culture War issues, exhibited a greater desire to hear from like-minded versus unlike-minded voters. In these studies, we also ruled out the possibility that people do not want to hear from their opponents because they already know a lot about them. Finally, we found that people on the left and the right are motivated to avoid hearing from the other side for some of the same reasons: the anticipation of cognitive dissonance and the undermining of a fundamental need for a shared reality with other people.

\section{Limitations of the Present Studies}

The present studies had some noteworthy limitations. First, the samples studied were recruited from a crowdsourcing website; the degree to which these samples are representative of the population is unknown. Although our samples spanned most of the lifespan and represented the male and female genders, they tended to financially well off, well-educated, and predominantly White. The restriction of North American samples further limited the generalizability of the findings. Future research should investigate whether desires to avoid crosscutting information is ideologically symmetric with representative samples and in a variety of countries.

Second, political ideology is multifaceted: people can independently hold liberal or conservative views on social issues (e.g., for or against same-sex marriage), economic issues (e.g., higher or lower taxes), and foreign policy (e.g., for or against war). The focus of the present studies was social issues. Future research should investigate whether the motivation to remain within one's ideological bubble is ideologically symmetric on economic and foreign policy issues. 
Third, all of the opinions in our studies were pre-existing. This introduces a correlational element to all of our studies, which draws in possible confounds (e.g., memory of the history of debates on an issue). Future research might experimentally induce participants to form a new opinion on a novel or hypothetical issue to assess whether liberals or conservatives are more motivated to avoid opponents' opinions with fewer confounds.

A fourth possible limitation to the present research is that the contexts we presented were inherently asymmetric. Perhaps one side has better reasons to want to ignore the other side. Liberals may think that conservatives' views truly are irrational, indefensible, and the product of fear-and thus not worth hearing. However, a conservative reader might have a similar impression of liberals' beliefs, opinions, and reasoning.

Although the present data cannot directly rule out the possibility that one side's views are objectively less valid than the others', we generally favor the interpretation that both sides' views are based on bounded rationality. Both liberals and conservatives perceive themselves to be locked in a struggle between good and evil, wherein "we" are good and "they" are evil. Ideology breeds self-righteousness - the sense that "our" side is motivated by love and reason and the “other" side is motivated by hatred and ignorance (Skitka \& Bauman, 2008; Waytz et al., 2014). We also acknowledge that the reasonableness of the two sides might sometimes be verifiably asymmetric. For example, during the 2016 U.S. presidential election, Donald Trump (Republican) lied much more often than did his Democratic opponent, Hillary Clinton (Comparing Hillary Clinton, Donald Trump on the Truth-O-Meter, n.d.). Additionally, the emergence of fake news aimed at undermining the credibility of Clinton was another asymmetry in the defensibility of the two sides. Even under these circumstances, listening to the other side 
could help individuals prepare counterarguments, and have a disarming effect, both of which may foster meaningful and respectful dialog.

\section{Conclusion}

College students and administrators on both sides of the Culture War have attempted to and succeeded at suppressing free speech on campus when it conflicted with their own beliefs. For example, Condoleeza Rice was scheduled to deliver a commencement speech at Rutgers University; she withdrew in response to liberal student protests about her role in the George W. Bush administration's position on torture (Sguelglia, 2014). And Vicki Kennedy (wife of the late Senator Ted Kennedy) was disinvited to give a commencement speech at Anna Maria College (a Catholic institution) in 2012 because of objections to her liberal views on social issues, such as abortion and gay marriage (Kabas, 2014). These anecdotes may be revealing of a banal desire that ideologically minded people have to avoid listening to people with opposing ideals. The result of this desire to avoid ideological incongruous views is that liberals and conservatives live in ideological information bubbles, and what could ultimately be a contest of ideas is being replaced by two, non-interacting monopolies. 


\section{References}

Bakshy, E., Messing, S., \& Adamic, L. A. (2015). Exposure to ideologically diverse news and opinion on Facebook. Science, 348(6239), 1130-1132. doi:10.1126/science.aaa1160

Barberá, P., Jost, J. T., Nagler, J., Tucker, J. A., \& Bonneau, R. (2015). Tweeting from left to right: Is online political communication more than an echo chamber? Psychological Science, 26(10), 1531-1542. doi:10.1177/0956797615594620

Brandt, M. J., Chambers, J. R., Crawford, J. T., Wetherell, G., \& Reyna, C. (2015). Bounded openness: The effect of openness to experience on intolerance is moderated by target group conventionality. Journal of Personality and Social Psychology, 109(3), 549-568. doi:10.1037/pspp0000055

Brandt, M. J., Reyna, C., Chambers, J. R., Crawford, J., \& Wetherell, G. (2014). The ideological conflict hypothesis: Intolerance among both liberals and conservatives. Current Directions in Psychological Science, 23, 27-34. doi:10.1177/0963721413510932

Chambers, J., Schlenker, B., \& Collisson, B. (2013). Ideology and prejudice: The role of value conflicts. Psychological Science, 24, 140-149. doi:10.1177/0956797612447820

Collins, T. P., Crawford, J. T., \& Brandt, M. J. (2015). Ideological symmetry in people’s avoidance of dissonance-arousing situations: A failure to closely or conceptually replicate Nam, Jost, and Van Bavel (2013). Manuscript in preparation.

Comparing Hillary Clinton, Donald Trump on the Truth-O-Meter (n.d.). Retrieved from http://www.politifact.com/truth-o-meter/lists/people/comparing-hillary-clinton-donaldtrump-truth-o-met/ 
Conway, L. G., Gornick, L. J., Houck, S. C., Anderson, C., Stockert, J., Sessoms, D., \& McCue, K. (2015). Are conservatives really more simple- minded than liberals? The domain specificity of complex thinking. Political Psychology, doi:10.1111/pops.12304

Crawford, J. T., Brandt, M. J., Inbar, Y., Chambers, J., \& Motyl, M. (2016). Social and economic ideologies differentially predict prejudice across the political spectrum, but social issues are most divisive. Journal of Personality and Social Psychology.

Echterhoff, G., Higgins, E. T., \& Levine, J. M. (2009). Shared reality: Experiencing commonality with others' inner states about the world. Perspectives on Psychological Science, 4(5), 496-521. doi:10.1111/j.1745-6924.2009.01161.x

Festinger, L. (1957). A theory of cognitive dissonance. Stanford University Press.

Frimer, J. A., Biesanz, J. C., Walker, L. J., \& MacKinlay, C. W. (2013). Liberals and conservatives rely on common moral foundations when making moral judgments about influential people. Journal of Personality and Social Psychology, 104, 10401059. doi:10.1037/a0032277

Frimer, J. A., Gaucher, D. \& Schaefer, N. K. (2014). Political conservatives’ affinity for obedience to authority is loyal, not blind. Personality and Social Psychology Bulletin, 40, 1205-1214. doi: 10.1177/0146167214538672

Frimer, J. A., Motyl, M., \& Tell, C. E. (2017). Sacralizing liberals and fair-minded conservatives: Ideological symmetry in the moral motives in the Culture War. Analyses of Social Issues and Public Policy. doi: 10.1111/asap.12127

Frimer, J. A., Tell, C. E. \& Haidt, J. (2015). Liberals condemn sacrilege too: The harmless desecration of Cerro Torre. Social Psychological and Personality Science, 6, 878886. doi: $10.1177 / 1948550615597974$ 
Gruenfeld, D. H. (1995). Status, ideology, and integrative complexity on the U.S. Supreme Court: Rethinking the politics of political decision making. Journal of Personality and Social Psychology, 68, 5-20. doi:10.1037/0022-3514.68.1.5

Harrington, J. R., \& Gelfand, M. J. (2014). Tightness-looseness across the 50 United States. PNAS Proceedings of the National Academy of Sciences oft United States of America, 111(22), 7990-7995. doi:10.1073/pnas.1317937111

Hart, W., Albarracín, D., Eagly, A. H., Brechan, I., Lindberg, M. J., \& Merrill, L. (2009). Feeling validated versus being correct: A meta-analysis of selective exposure to information. Psychological Bulletin, 135(4), 555-588. doi:10.1037/a0015701

Hedges, L.V., Vevea, J.L. (1998). Fixed- and random-effects models in meta-analysis. Psychological Methods, 3, 486-504. doi: 1082-989X/98/53.00

Iyengar, S., \& Hahn, K. S. (2009). Red media, blue media: Evidence of ideological selectivity in media use. Journal of Communication, 59(1), 19-39. doi:10.1111/j.14602466.2008.01402.x

Iyengar, S., Hahn, K. S., Krosnick, J. A., \& Walker, J. (2008). Selective exposure to campaign communication: The role of anticipated agreement and issue public membership. The Journal of Politics, 70(1), 186-200. doi:10.1017/S0022381607080139

Jost, J. T., Glaser, J., Kruglanski, A. W., \& Sulloway, F. J. (2003). Political conservatism as motivated social cognition. Psychological Bulletin, 129(3), 339-375. doi:10.1037/00332909.129.3.339

Jost, J. T., Nosek, B. A., \& Gosling, S. D. (2008). Ideology: Its resurgence in social, personality, and political psychology. Perspectives on Psychological Science, 3(2), 126-136. doi:10.1111/j.1745-6916.2008.00070.x 
Kabas, M. (2014). Pomp and protest: 11 controversial college commencement speakers.

Retrieved from http://www.today.com/news/pomp-protest-11-controversial-collegecommencement-speakers-2D79672771

Kunda, Z. (1990). The case for motivated reasoning. Psychological Bulletin, 108(3), 480-498. doi:10.1037/0033-2909.108.3.480

Lazarsfeld, P. F., Berelson, B., \& Gaudet, H. (1944). The people's choice. Oxford, England: Duell, Sloan \& Pearce.

McCrae, R. R. (1996). Social consequences of experiential openness. Psychological Bulletin, 120(3), 323-337. doi:10.1037/0033-2909.120.3.323

Nam, H. H., Jost, J. T., \& Van Bavel, J. J. (2013). "Not for All the Tea in China!” Political Ideology and the Avoidance of Dissonance-Arousing Situations. PloS One, 8(4), e59837.

Nickerson, R. S. (1998). Confirmation bias: A ubiquitous phenomenon in many guises. Review of General Psychology, 2(2), 175-220. doi:10.1037/1089-2680.2.2.175

Onraet, E., Van Hiel, A., Dhont, K., Hodson, G., Schittekatte, M., \& De Pauw, S. (2015). The association of cognitive ability with right- wing ideological attitudes and prejudice: A meta- analytic review. European Journal of Personality, 29(6), 599-621. doi:10.1002/per.2027

Overack, L. (1941). Campaign finance in the presidential election of 1940. The American Political Science Review, 35 (4), 701-727. doi: 10.2307/1948076

Sears, D. O., \& Freedman, J. L. (1967). Selective exposure to information: A critical review. Public Opinion Quarterly, 31(2), 194-213. doi:10.1086/267513

Sguelglia, K. (2014). Condoleezza Rice declines to speak at Rutgers after student protests. Retrieved from http://www.cnn.com/2014/05/04/us/condoleeza-rice-rutgers-protests/ 
Sibley, C. G., \& Duckitt, J. (2008). Personality and prejudice: A meta-analysis and theoretical review. Personality and Social Psychology Review, 12(3), 248-279. doi:10.1177/1088868308319226

Skitka, L. J., \& Bauman, C. W. (2008). Moral conviction and political engagement. Political Psychology, 29(1), 29-54. doi:10.1111/j.1467-9221.2007.00611.x

Smith, S. M., Fabrigar, L. R., \& Norris, M. E. (2008). Reflecting on six decades of selective exposure research: Progress, challenges, and opportunities. Social and Personality Psychology Compass, 2(1), 464-493. doi:10.1111/j.1751-9004.2007.00060.x

Stroud, N. J. (2008). Media use and political predispositions: Revisiting the concept of selective exposure. Political Behavior, 30(3), 341-366. doi:10.1007/s11109-007-9050-9

Talhelm, T., Haidt, J., Oishi, S., Zhang, X., Miao, F. F., \& Chen, S. (2015). Liberals think more analytically (more 'WEIRD') than conservatives. Personality and Social Psychology Bulletin, 41(2), 250-267. doi:10.1177/0146167214563672

Vail, K. I., \& Motyl, M. (2010). Support for diplomacy: Peacemaking and militarism as a unidimensional correlate of social, environmental, and political attitudes. Peace and Conflict: Journal of Peace Psychology, 16(1), 29-57. doi:10.1080/10781910903486813

Waytz, A., Young, L. L., \& Ginges, J. (2014). Motive attribution asymmetry for love vs. hate drives intractable conflict. PNAS Proceedings of The National Academy of Sciences of the United States of America, 111(44), 15687-15692. doi:10.1073/pnas.1414146111

Webb, T. L., Chang, B. I., \& Benn, Y. (2013). 'The ostrich problem': Motivated avoidance or rejection of information about goal progress. Social and Personality Psychology Compass, 7(11), 794-807. doi:10.1111/spc3.12071 